\title{
Short-Chain Fatty Acids Induce Colonic Mucosal Injury in Rats with Various Postnatal Ages
}

\author{
SUHAS M. NAFDAY, WEI CHEN, LUYING PENG, MARK W. BABYATSKY, \\ IAN R. HOLZMAN, AND JING LIN \\ Departments of Pediatrics [S.M.N., W.C., L.P., I.R.H., J.L.] and Medicine [M.W.B.], Mount Sinai School \\ of Medicine, New York, NY 10029
}

\begin{abstract}
Short chain fatty acids (SCFAs) may play a role in the pathogenesis of neonatal necrotizing enterocolitis. To evaluate the injurious effect of SCFAs on the colonic mucosa of rats at various postnatal developmental stages, we studied a total of 170 newborn Sprague-Dawley rats at postnatal ages days 3, 9, and 23. A 1.8-F silastic catheter or umbilical catheter was inserted rectally deep into the proximal colon of the rats. Rats from each of the three postnatal age groups were randomly divided to receive one of the following distinct SCFA solutions: acetic acid, butyric acid, propionic acid, or a mixture of above SCFAs solutions. An additional subgroup of rats from each of the age groups received normal saline as a control. The concentration of each SCFA solution was $300 \mathrm{mM}$, and the $\mathrm{pH}$ of all solutions was adjusted to 4.0. The volume of administered solution was 0.1 $\mathrm{mL} / 10 \mathrm{~g}$ of body weight. After $24 \mathrm{~h}$, all rats were killed and the daily weight change was recorded and proximal colon was collected for histologic examination. A histologic injury score
\end{abstract}

\section{ABSTRACT}

was used to quantify the severity of mucosal injury. The severity of mucosal injury induced by luminal SCFAs administration decreased as the rats matured; by postnatal day 23 , the injury caused by SCFAs was minimal. Thus, the severity of the colonic mucosal injury induced by luminal SCFAs is maturation dependent; the immature state of the mucosal defense in early postnatal age in newborn rat may explain its greater vulnerability to luminal SCFAs. (Pediatr Res 57: 201-204, 2005)
$\mathbf{A A}$, acetic acid
BA, butyric acid
NEC, necrotizing enterocolitis
NS, normal saline
PA, propionic acid
SCFA, short chain fatty acid

Abbreviations
Neonatal necrotizing enterocolitis (NEC), an enigmatic disease, still is one of the major causes of morbidity and mortality in premature infants. Despite many years of investigation, the pathogenesis of NEC remains elusive. Prematurity, formula feeding, and bacterial colonization have been widely recognized as three major risk factors for the pathogenesis of NEC (1). It has been hypothesized that multiple factors predispose the premature infant to intestinal mucosal injury and necrosis as a result of activation of the inflammatory cascade (2).

NEC occurs mainly in premature infants. Immaturity of the intestinal mucosa may contribute to the higher incidence of NEC in premature infants. Short-chain fatty acids (SCFAs), products of

Received March 10, 2004; accepted June 10, 2004

Correspondence: Jing Lin, M.D., Jack \& Lucy Clark Department of Pediatrics, Mount Sinai School of Medicine, One Gustave L. Levy Place, Box 1508, NY, NY 10029-6574; e-mail: jing.lin@mssm.edu

Supported by National Institutes of Health Grant 1-K08-HD-01223 and the Educational Foundation of America.

Presented in part at the annual meeting of the Pediatric Academic Society, Seattle, WA, May 3-6, 2003.

S.H.N.'s current affiliation is the Department of Pediatrics, Albert Einstein College of Medicine/Children's Hospital at Montefiore, Bronx, NY 10461.

DOI: 10.1203/01.PDR.0000150721.83224.89 bacterial fermentation in the intestinal lumen, have been proposed to play a key role in the pathogenesis of NEC $(3,4)$. We showed previously that acetic acid and butyric acid, two of the main SCFAs, can induce concentration- and $\mathrm{pH}-$ dependent intestinal mucosal injury in newborn rats (5). We have suggested that accumulation of SCFAs in the proximal colon as a result of carbohydrate maldigestion and/or bacterial overgrowth may lead to intestinal mucosal injury in premature infants $(3,5)$. Concentrations of SCFAs in distal ileum may also increase because of reflux through the ileocecal valve and/or local bacterial overgrowth $(6,7)$. This hypothesis can explain the major risk factors for the development of NEC and pathology seen most commonly in the proximal colon and distal ileum $(1,8)$. The aim of this study was to examine further the injurious effect of SCFAs on colonic mucosa of rats at various maturation stages and to determine whether postnatal maturational changes in intestine can modify the injurious effects of SCFAs on intestinal mucosa.

\section{METHODS}

A total of 170 newborn Sprague-Dawley rats in three different age groups (3-4, 9-10, and 23-24 d of life) were studied. Newborn rats (Taconic 


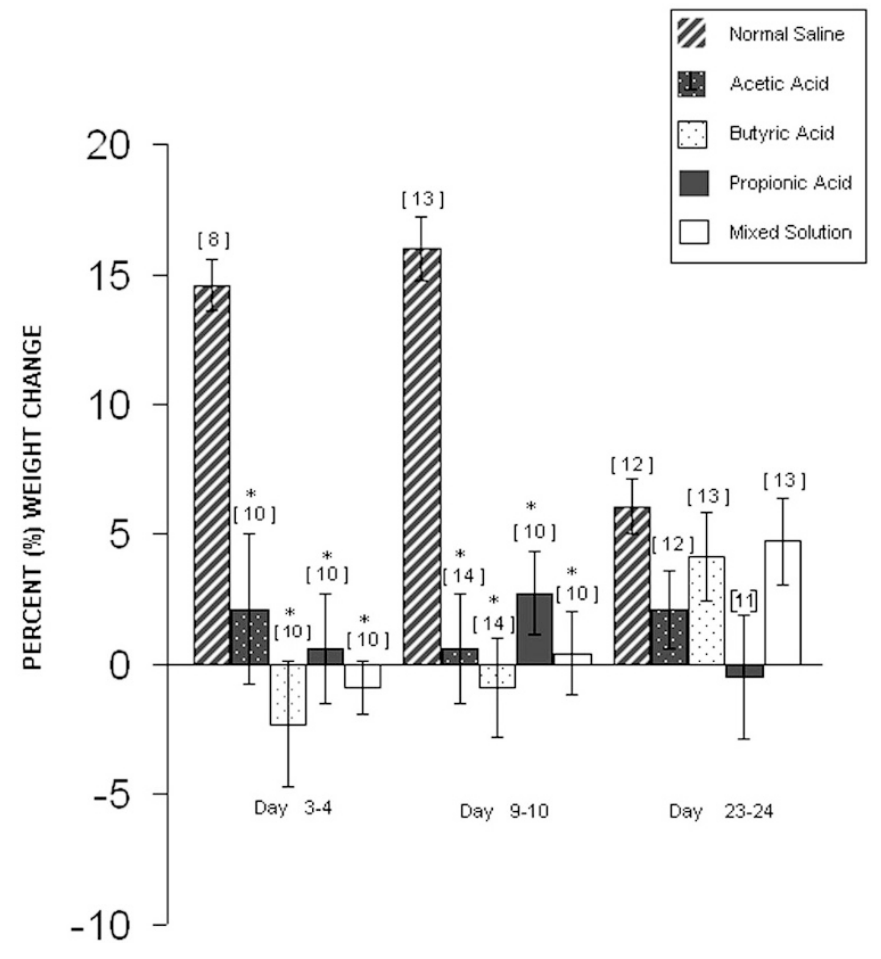

Figure 1. Percentage body weight changes at $24 \mathrm{~h}$ after luminal administration of SCFAs or NS. The numbers in parentheses represent the sample numbers in that subgroup. The rats that received SCFAs had significantly impaired weight gain compared with the rats that received NS, especially in the day $3-4$ and day $9-10$ age groups $\left({ }^{*} p<0.01\right.$, ANOVA)

Laboratory, New York, NY) all were housed with their mothers before they were weaned at 21-22 d of age. Once weaned, they were fed ad libitum with standard rat diet with water freely available. All rats were maintained in alternate 12-h periods of light and darkness.

The study protocol was similar to the one we have described previously (5). In this study, all three main SCFAs [acetic acid (AA), butyric acid (BA), and propionic acid (PA); Sigma Chemical Co., St. Louis, MO] in a concentration of $300 \mathrm{mM}$ (pH 4.0) and a mixture of SCFAs $(180 / 60 / 60 \mathrm{mM}$ of AA/BA/PA, $\mathrm{pH} 4.0$ ) were used. The volume administered for each rat was at $0.1 \mathrm{~mL} / 10 \mathrm{~g}$ of body weight, and the final osmolarity was $\sim 340 \mathrm{mOsmol} / \mathrm{L}$ for all SCFA solutions. Normal saline ( $\mathrm{pH}$ adjusted to 4.0 with $\mathrm{HCl}$ ) was administered at the same volume to a subgroup of rats in each age group as a control. Therefore, there were five subgroups in each age group. Littermates were usually assigned to different subgroups. A 1.9-F silastic catheter (Becton Dickinson, Sandy, UT) was used in 3- to 4-d-old rats, a 3.5-F regular umbilical catheter was used for 9- to 10-d-old rats, and a 5.0-F umbilical catheter was used in 23- to 24-d-old rats. The catheter was inserted deep into the proximal colon for intraluminal administration of SCFA solutions or normal saline (NS). The administration of the SCFA solutions was not blinded to the investigators because of their obvious odor. In each individual experiment, various SCFA solutions were administered into different subgroups on the same day. Animals were housed with and cared for by their mothers before and after the experiments except for the 23- yo 24-d-old age group.

Twenty-four hours after the luminal administration of the SCFA solutions or NS, the rats were weighed and killed by cervical dislocation. The proximal colon was removed, fixed in $4 \%$ neutral phosphate-buffered formalin, crosssectioned at 2-mm intervals, and embedded in paraffin. A 5- $\mu \mathrm{m}$-thick section from each block was stained with hematoxylin and eosin. Two investigators who were blinded to the group assignment evaluated all of the slides, and a histologic injury score was assigned to all of the tissues. We used the same scoring system as previously described $(5,9)$. Briefly, scores range from 0 to 5 on the basis of the degree of the injury: 0 for normal histology, 1 for minimal to mild inflammation without necrosis, 2 for moderate to severe inflammation without necrosis, 3 for mucosal necrosis and/or distorted/regenerative archi- tecture indicative of previous severe epithelial injury, 4 for mucosal and submucosal necrosis, and 5 for transmural necrosis or perforation.

The Institutional Animal Care and Use Committee approved the research protocol. The data are presented as mean values and SEM. ANOVA was used for statistical analysis. The difference between each group was compared with the Tukey test or the Kruskal-Wallis test when the data were not normally distributed, and $p<0.05$ was considered statistically significant.

\section{RESULTS}

The baseline body weights were not different $(p>0.05)$ among all five subgroups in each age group. The percentage change in body weight at $24 \mathrm{~h}$ in all subgroups from each age group is shown in Fig. 1. In the day 3-4 group, the weight change in the NS subgroup was $14.7 \pm 1.0 \%$ (mean \pm SEM), whereas the rats that received each individual SCFA or the mixture of SCFA either had a minimal weight gain or lost weight $(p<0.001)$. The same results were seen in the day $9-10$ group $(p<0.001)$. However, by $23-24 \mathrm{~d}$ of age, normal weight gain was much less. The rats that received NS gained $\sim 6.1 \pm 1.1 \%$ of body weight in $24 \mathrm{~h}$. The differences in percentage weight changes among all five subgroups in the 23to 24-d age group were not statistically significant $(p>0.05)$.

At the time the rats were killed, almost all of the rats in the day 3-4 group that received each individual SCFA or SCFA mixture had either isolated perforation in the proximal colon or severe necrosis in the colon and distal ileum. The tissues were friable and grossly hemorrhagic. Peritonitis was evident in rats with perforations. None of control rats that received NS had perforations. In the day 9-10 age group, the rats that received SCFA had grossly evident intestinal edema and patchy hemorrhage, especially in the proximal colon and distal ileum. The injury pattern and degree in rats that received each individual SCFA or mixture of SCFAs were similar. The gross examination of colonic tissues from 23- to 24-d-old animals that had received SCFAs either looked completely normal or had minimal erythema and mild edema.

In the day 3-4 and day 9-10 groups, histologic examination revealed that the colonic tissues from the animals that received individual SCFA or a mixture of SCFAs had severe necrosis, with the depth of involvement usually severe. Transmural necrosis was common and was usually associated with intense neutrophilic infiltration. In the day 23-24 age group, the majority of animals that received either individual SCFA or a mixture of SCFAs had intact colonic tissues or had minimal focal mucosal necrosis with scattered inflammatory cells. All colonic tissues from the NS control group in all three age categories were normal. The mean microscopic colonic tissue injury scores are presented in Fig. 2. In each age group, the mucosal injuries induced by individual SCFA or by the mixture of SCFAs were not different $(p>0.05)$. However, the severity of injury decreased in older animals, especially in the day 23-24 group $(p<0.01)$. Representative histologic slides are shown in Fig. 3.

\section{DISCUSSION}

The proximal-to-distal passage of undigested carbohydrates across the ileocecal valve occurs normally throughout life (10). In newborn infants, normal intestinal bacterial colonization is 


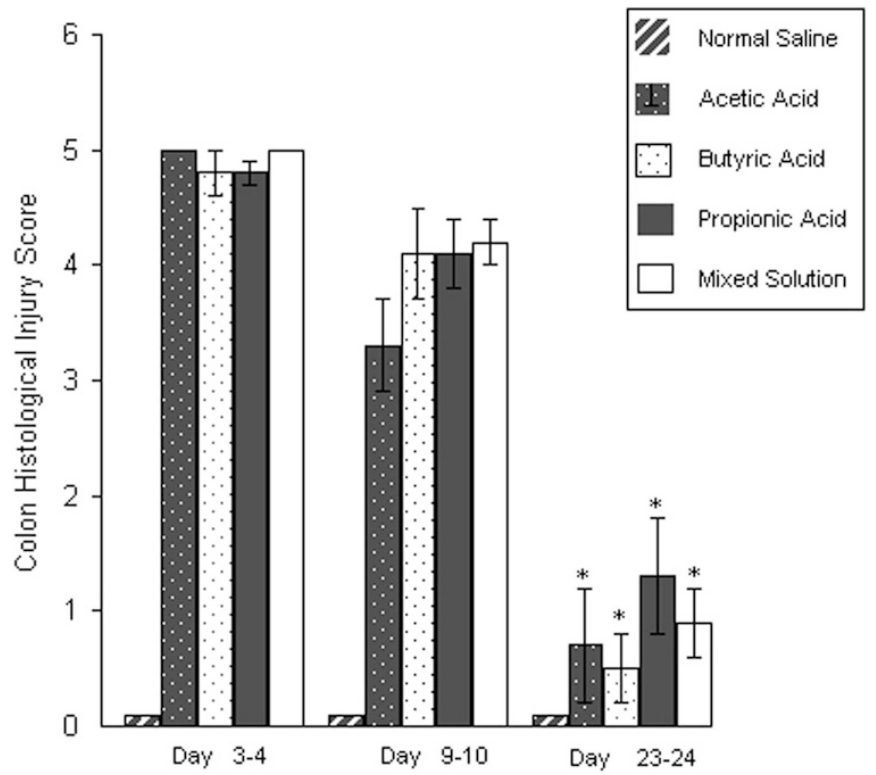

Figure 2. The mean histologic injury scores of proximal colon of rats from each group. The severity of each individual SCFA or the mixture of SCFAinduced mucosal injury decreased as the rats matured $\left({ }^{*} p<0.01\right.$, ANOVA). Within each age group, the differences in injury scores among the each individual SCFA or the SCFA mixture subgroups were not statistically significant.

established once enteral feeding is advanced. In the anaerobic environment of the colon, bacteria rapidly ferment carbohydrates to gases (hydrogen, carbon dioxide, and, in some cases, methane) and SCFAs (11). This process of bacterial fermentation of undigested carbohydrates plays an important role in normal intestinal biology such as water and salt absorption in the colon, energy salvation, and colonic mucosal maturation $(4,11)$. Because of a relative lactase deficiency in the small intestine, a significant amount of lactose ingested by normal premature infants may be fermented into SCFAs and then
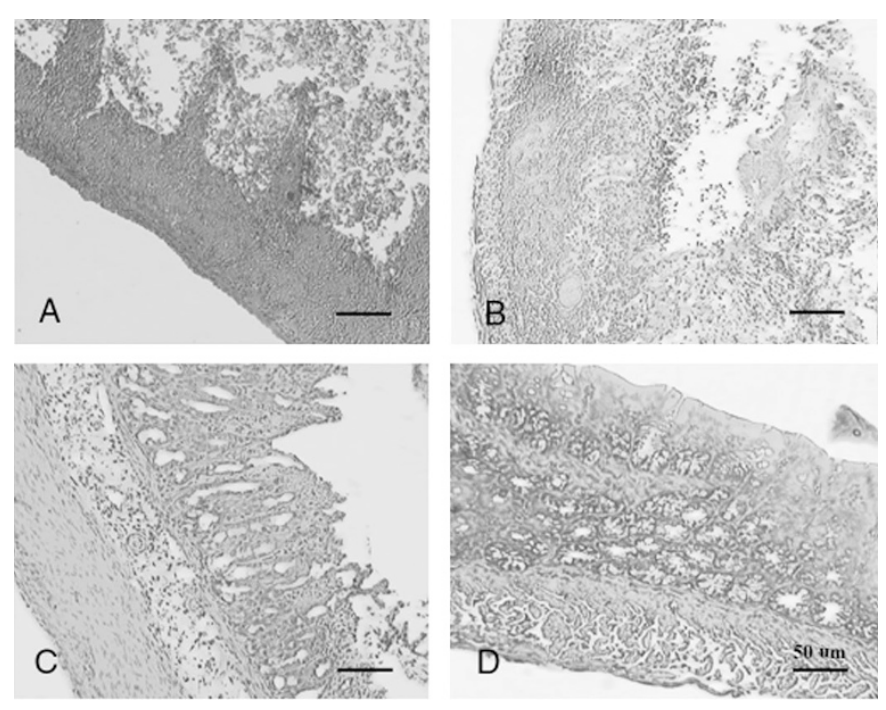

Figure 3. Representative histology of the colon from rats in different groups. (A) Colon of a 4-d-old rat that received the mixture of SCFAs. $(B)$ Colon of a 10-d-old rat that received the mixture of SCFAs. (C) Colon of a 24-d-old rat that received the mixture of SCFAs. $(D)$ Colon of a 24-d-old rat that received NS control. Magnification: $\times 100$ absorbed $(12,13)$. However, in some premature infants, an abnormal state of SCFA overproduction may arise during periods of significant carbohydrate malabsorption and/or bacterial overgrowth, which may exceed the buffering and absorptive capacity of the colon and lead to an increased concentration of SCFAs in the colon $(13,14)$. Levels of SCFAs in the distal ileum may also increase from backward reflux across the ileocecal valve or as a result of local bacterial overgrowth. High concentrations of SCFAs can significantly lower the $\mathrm{pH}$ in the bowel $(15,16)$.

We showed previously that the luminal administration of AA or BA can cause intestinal mucosal injury in 9- to 10-d-old newborn rats, and mild mucosal injury can be induced by AA or BA at a concentration as low as $150 \mathrm{mM}$ (5). In this study, we further demonstrated that PA, another major SCFA, and the mixture of SCFAs can cause similar injury to the colon once administered locally. Furthermore, we demonstrated that SCFA-induced mucosal injury is maturation dependent; postweaned rats are more resistant to the injury induced by the luminal administration of high concentrations of SCFAs. The mechanism for this injurious effect of SCFAs on intestinal mucosa is not clear. SCFAs in very high concentrations have been used to produce a reliable colitis model in adult rodents, and this effect could not be produced by low $\mathrm{pH}$ alone or by the butyrate anion at neutral or alkaline $\mathrm{pH}(17)$. The chemical property of SCFAs may be responsible for the injurious effect of SCFAs on intestinal mucosa. At $\mathrm{pH} 4.0$, which was chosen on the basis of the observation of Clark et al. (15) that 16 of 17 patients with NEC had intraluminal contents with a $\mathrm{pH}$ of $<5(\mathrm{pH}$ range, 3.8-4.6), most of the SCFAs (weak organic acids) are in free acid form and are most likely absorbed into intestinal mucosal cells via diffusion (18). The osmolarity of each SCFA solution was similar and within the isotonic range; furthermore, in our previous study, using $300 \mathrm{mM}$ of lactic acid at $\mathrm{pH} 4.0$ with the final osmolarity increased to $500 \mathrm{mOsmol} / \mathrm{L}$ did not induce any identifiable colonic mucosal injury in 9- to 10-d-old newborn rats (5). Therefore, the osmolarity alone is unlikely to explain the injurious effect of SCFAs on colonic mucosa.

Whether endogenous luminal SCFAs in premature infants can increase to a concentration that is toxic to the intestinal mucosa remains to be proved. In humans, the normal physiologic concentration of total SCFAs ranges from 70 to 140 $\mathrm{mM}$ in the proximal colon to 20 to $70 \mathrm{mM}$ in the distal colon (19-21). The concentration of total SCFAs may reach a potentially toxic $200-300 \mathrm{mM}$ in premature infants when carbohydrates are poorly digested and/or gastrointestinal motility is abnormal. The ratio of the mixture of SCFAs used in this study is similar to the prevailing concentrations that exist in the proximal colon and causes an injury pattern similar to each individual SCFA. This is evidence further supporting the concept that an increased concentration of total SCFAs can lead to the development of NEC in premature infants.

In comparison with the newborn human infant, the gastrointestinal tract in newborn rats is relatively immature. There are significant normal structural and functional changes in the 
intestinal tract during the first few weeks of life, especially at the time of weaning (22-24). Some factors that are important in maintaining the intestinal mucosal barrier function also develop postnatally $(25,26)$. The mechanism for maturation changes in the colon associated with weaning is still poorly understood (22). In our study, we demonstrated that the severity of mucosal injury induced by luminal SCFA administration decreased with age, suggesting that the colonic mucosa in a postweaning rat is more resistant to the injurious effect of SCFAs. Because all other confounding factors such as the dosage and the method of administration in the experiments were controlled, the difference in the vulnerability of the colonic mucosa to SCFAs is best explained by the degree of mucosal maturation.

On the basis of our data, we speculate that overproduction/ accumulation of luminal SCFAs in premature infants may play a key role in the pathogenesis of NEC. In preterm infants, before enteral feeding is introduced, the colon is relatively devoid of bacterial colonization. Therefore, even though the intestinal mucosa is vulnerable to SCFA injury, the incidence of NEC is very low because there is limited SCFA production. Subsequently, as enteral feedings are advanced and the intestine is colonized with bacteria, the presence of undigested carbohydrate may lead to excessive SCFA production. If SCFAs cannot be absorbed or cleared promptly as a result of poor gastrointestinal motility or a decreased blood flow to the intestines, then increased concentration of SCFAs may result in injury to the vulnerable intestinal mucosa of premature infants, a disease that we call NEC. Because the normal production of SCFAs in intestine is critical for intestinal adaptation and maturation (11,20), withholding enteral feeds to delay the normal bacterial colonization may not be a good option. More studies are needed to explore further the roles of SCFAs in the regulation of intestinal mucosal maturation and the pathogenesis of NEC.

\section{REFERENCES}

1. Neu J 1996 Necrotizing enterocolitis: the search for a unifying pathogenic theory leading to prevention. Pediatr Clin North Am 43:409-432

2. Hsueh W, Caplan MS, Qu XW, Tan XD, DePlaen IG, Gonzalez-Crussi F 2002 Neonatal necrotizing enterocolitis: clinical considerations and pathogenetic concepts. Pediatr Dev Pathol 6:6-23
3. Lin J 2004 Too much short chain fatty acids cause neonatal necrotizing enterocolitis. Med Hypotheses 62:291-293

4. Kien CL 1990 Colonic fermentation of carbohydrate in the premature infant: possible relevance to necrotizing enterocolitis. J Pediatr 117:S52-S58

5. Lin J, Nafday SM, Chauvin SN, Magid MS, Pabbatireddy S, Holzman IR, Babyatsky MW 2002 Variable effects of short chain fatty acids and lactic acid in inducing intestinal mucosal injury in newborn rats. J Pediatr Gastroenterol Nutr 35:545-550

6. Hoverstad T, Bjorneklett A, Fausa O, Midtvedt T 1985 Short-chain fatty acids in the small-bowel bacterial overgrowth syndrome. Scand J Gastroenterol 20:492-499

7. Lloyd DR, Brown JD, Brown GA, Booth IW 1992 Elevated short chain fatty acid concentrations in anaerobic small bowel contamination. Acta Paediatr 81:51-56

8. Ballance WA, Dahms BB, Shenker N, Kliegman RM 1990 Pathology of neonatal necrotizing enterocolitis: a ten year experience. J Pediatr 117:S6-S13

9. Nafday SM, Green RS, Chauvin SN, Holzman IR, Magid MS, Lin J 2002 Vitamin A supplementation ameliorates butyric acid induced intestinal mucosal injury in newborn rats. J Perinat Med 30:121-127

10. Bond JH, Levitt MD 1976 Fate of soluble carbohydrate in the colon of rats and man. J Clin Invest 57:1158-1164

11. Murray R 1990 Effects of bacterial fermentation end products on intestinal function: implications for intestinal dysfunction. J Pediatr 117:S59-S63

12. Kien CL, McClead RE, Cordero L Jr 1996 In vivo lactose digestion in premature infants. Am J Clin Nutr 64:700-705

13. MacLean WC Jr, Fink BB 1980 Lactose malabsorption by premature infants: magnitude and clinical significance. J Pediatr 97:383-388

14. Kien CL 1996 Digestion, absorption, and fermentation of carbohydrates in the newborn. Clin Perinatol 23:211-228

15. Clark DA, Thompson JE, Weiner LB, McMillan JA, Scneider AJ, Rokahr JE 1985 Necrotizing enterocolitis: intraluminal biochemistry in human neonates and a rabbit model. Pediatr Res 19:919-921

16. Brown RL, Gibson JA, Sladen GE, Hicks B, Dawson AM 1974 Effects of lactulose and other laxatives on ileal and colonic $\mathrm{pH}$ as measured by a radiotelemetry device. Gut 15:999-1004

17. McCafferty DM, Zeitlin IJ 1989 Short chain fatty acid induced colitis in mice. Int J Tissue React 11:165-168

18. Charney An, Micic L, Egnor RW 1998 Nonionic diffusion of short-chain fatty acids across rat colon. Am J Physiol 274:G518-G524

19. Cummings JH, Pomare EW, Branch WJ, Naylor CPE, MacFarlane GT 1987 Short chain fatty acids in human large intestine, portal hepatic and venous blood. Gut 28:1221-1227

20. Velazquez OC, Lederer HM, Rombeau JL 1997 Butyrate and the colonocyte. Production, absorption, metabolism, and therapeutic implications. Adv Exp Med Biol 427:123-134

21. Saemann MD, Bohmig GA, Zlabinger GJ 2002 Short chain fatty acids: bacterial mediators of a balanced host-microbial relationship in human gut. Wien Klin Wochenschr 114:289-300

22. Babyatsky MW, Podolsky DK 2003 Growth and development of the gastrointestinal tract. In: Yamada T, Alpers DH, Kaplowitz N, Laine L, Owyang C, Powell DW (eds) Textbook of Gastroenterology. Lippincott Williams \& Wilkins, Philadelphia, pp $521-556$

23. Finkel Y, Larsson L 1987 Morphometric and functional studies of proximal and distal colon in young and adult rats. J Pediatr Gastroenterol Nutr 6:454-459

24. Menard D, Dagenais P, Calvert R 1994 Morphological changes and cellular proliferation in mouse colon during fetal and postnatal development. Anat Rec 238:349359

25. Ouellette AJ Bevins CL 1999 Development of innate immunity in the small intestine. In: Sanderson IR, Walker WA (eds) Development of the Gastrointestinal Tract. BC Decker, Hamilton, pp 147-164

26. Lin J, Holzman IR, Jiang P, Babyatsky MW 1999 Expression of intestinal trefoil factor during rat intestinal development. Biol Neonate 76:92-97 